PLANT PATHOLOGY

Disease Resistance

from a Correspondent

A Discussion meeting on disease resistance in plants was held on December 9 at the Royal Society. Professor P. W. Brian (Cambridge), recalled in his opening remarks that the last meeting organized by the Royal Society on this subject had been in December 1947. The speakers on that occasion had been Professors W. Brown and F. T. Brooks, together with Mr F. C. Bawden. A link between these two meetings was provided by Sir Frederick Bawden as chair. man of the afternoon session.

In his introduction Professor R. K. S. Wood (Imperial College, London) had time to say something more particular about the hypersensitive reaction of plants to invasion by bacterial and fungal pathogens and about the phytoalexin response. His former colleague at the Imperial College, $\mathrm{Dr} \mathrm{B}$. J. Deverall (Agricultural Research Council at Wye College), took up the story of phytoalexins in more detail. These protective substances are produced by plants in response to challenge by microorganisms and also to chemical injury; the possibility that phytoalexins can explain why most plants are resistant to invasion by most microorganisms accounts for the wide interest aroused by these plant chemicals. Wyerone acid has recently been identified by Dr Deverall and associates as a phytoalexin produced by the broad bean. From work with potato, the plant in which $K$. O. Müller first demonstrated the phytoalexin response, Dr D. D. Clarke (University of Glasgow) cited evidence for the view that virulent races of the potato-blight fungus inhibit the synthesis of phytoalexin by their host.

Professor A. Kelman and Dr L. Sequeira (University of Wisconsin, Madison), with a variety of examples, made the point that bacterial pathogens are a natural choice for the study of resistance responses by the host. Working with Pseudomonas solanacearum, which causes wilt of tobacco, they have shown that prior infiltration of leaves with heat-killed bacterial cells or their protein fractions will protect leaves later challenged by the living pathogen, and that this protective response can move out into untreated leaves. From studies on the virus-yellows complex of sugar beet, Dr G. E. Russell (Plant Breeding Institute, Trumpington) demonstrated the wide spectrum of resistance mechanisms against virus infection, including a useful degree of resistance to colonization of leaves by aphid vectors.

The increasing threat to wheat and barley from yellow-rust epidemics produced for Dr R. C. F. Macer (Rothwell

Plant Breeders Ltd, Lincoln) the most lively discussion of the day, though this did not detract from the promise of $F_{1}$ hybrid varieties as a new kind of answer to this problem. The last two papers in the afternoon served further to emphasize the central theme of this meeting, that is, the wide variety of diseaseresistance mechanisms. Dr P. W. Talboys (East Malling Research Station) developed in detail his explanation of how the sequence of post-invasion events can account for the various responses of susceptibility, tolerance or resistance in plants invaded by vascularwilt fungi. Similarly, Dr J. Rishbeth (University of Cambridge) was able to explain some resistance mechanisms of trees, such as the resin response of conifers, that are effective against rootinfecting fungi.

The general impression left by this meeting was that few if any of the new developments in research described during the day were of secondary importance; to counter the genetic opportunism of evolving pathogens, plant pathologists would have to exploit to the full all the resistance mechanisms possessed by the plant.

\title{
Variation in Chromosome Pairing in Diploid Wheat
}

BrEAD wheat, Triticum aestivum, behaves as a classical allohexaploid $(2 n=6 x=42)$ with regular bivalent formation at meiosis and disomic inheritance, although the three genomes, A, B, and D, are sufficiently homologous for chromosome pairing to occur between them. This homoeologous pairing is prevented by an allele at the $P h$ locus on chromosome 5B. All of the synthetic tetraploid and hexaploid wheats produced from the probable diploid ancestors show homoeologous pairing, indicating that none of the diploid plants used has an allele which prevents this. On the contrary, all plants of Aegilops speltoides that have been studied suppress the control by the $P h$ allele. Aegilops speltoides is considered the most likely contributor of the $\mathrm{B}$ genome. Although it has been assumed that control by the $P h$ allele originated in the polyploid wheats, the origin and establishment of this genetic control of chromosome pairing is still an unsolved problem.

In Nature New Biology next week (January 12), Dover and Riley report the first evidence of genetic variation, in a diploid species, which influences homoeologous pairing in polyploid wheat. This variation is present in Aegilops mutica which, like Ae. speltoides, has previously been shown to suppress the activity of the $P h$ allele. Homoeologous pairing and chiasma frequency were studied in several $T$. aestivum $\times$ Ae. mutica progenies. The tetraploid hybrids had the A, B, and D
TUMOUR VIROLOGY

Mitosis Required

from our Cell Biology Correspondent

Although nobody has yet suggested a good reason why it should be the case, many tumour virologists maintain that the transformation of cells by both RNA and DNA tumour viruses depends on the cell going through at least one round of division, a quantal mitosis, after it has been infected. The idea has an immaculate pedigree, stemming from the experiments of such people as Green, Todaro and Temin to mention but a few, and as Weiss now points out (Virology, 46, 209; 1971) it ties in with investigations of the differentiation of myoblasts in culture which have led Holtzer and others to conclude that cellular transformation during normal differentiation depends on a quantal mitosis.

Most of the experiments with the RNA tumour viruses, which have been interpreted in favour of the quantal mitosis idea, have involved the use of drugs which inhibit either DNA replication or mitosis. What is notable about the

genomes from a homozygous $T$. aestivum and the $\mathrm{Mt}$ genome from naturally heterozygous Ae. mutica.

Dover and Riley counted the chiasmata in seven hybrid families, and found that the chiasma frequencies for the individual plants fell into four classes with mean chiasma frequencies of $1.51,4.13,7.06$ and 12.91 chiasmata per cell. In the lowest pairing class homoeologous pairing was prevented and there was a high frequency of univalents. In the highest pairing class bivalents, trivalents and quadrivalents were formed. The segregation into four pairing classes indicates that more than two alleles are involved, possibly two alleles at each of two loci. One family segregated $1: 1: 1: 1$, indicating heterozygosity at both loci in the parent plant.

The discovery in Ae. mutica of this variation in factors regulating chromosome pairing suggests that similar variation may be present in other diploid species. The effect of such variation in the diploids is not known, but if it is effective in hybrids lacking the $P h$ allele, it provides material for the immediate establishment of regular bivalent formation in the new spontaneous amphiploid, that is, a diploid with low pairing alleles would give a classical allopolyploid. The finding of this variation in a diploid provides evidence that the $P h$ allele need not have arisen at the tetraploid level. If similar variation exists in $A e$. speltoides it would add weight to the evidence that this was the donor of the $B$ genome of wheat. 
experiments Weiss has just reported in Virology is the way in which he blocked DNA synthesis and mitosis of the infected cells. Instead of exposing chick cells to an inhibitor he plated freshly trypsinized chick cells, infected with Rous sarcoma virus, onto confluent monolayers of either mouse cells or chick cells. When mouse cell monolayers are used the chick cells do not replicate their DNA or divide, neither do they develop into foci of transformants. By contrast the infected chick cells plated on top of chick cell monolayers do divide and do develop into foci without the virus replicating in the monolayer cells.

Weiss believes that these findings are consonant with the quantal mitosis idea and he has an equally fashionable explanation for the differential inhibition of chick cell division by monolayers of chick and mouse cells. He suggests, à la Burger, that the mouse cells elaborate and secrete some cell surface component which is stripped from chick cells when they are exposed to trypsin and which is required to keep the cells susceptible to those growth regulatory signals which act in dense cultures to inhibit cell division. Freshly trypsinized chick cells plated onto a monolayer of mouse cells pick up this surface component and their multiplication becomes inhibited. By contrast, Weiss envisages that monolayers of chick cells do not secrete this component.

Be that as it may, Weiss is now hunting mutant Rous viruses which are conditionally sensitive to the density dependent growth of their hosts. Kawai and Hanafusa (ibid., 470) who have also joined in the search for temperature sensitive mutants of Rous sarcoma virus have come up with one mutant Ts 68 which is more or less identical to the mutant isolated and described by Martin (Nature, 227, 1021; 1970). Both the Martin and the Kuwai and Hanafusa mutants appear to define a Rous sarcoma virus gene which specifies a protein that is not a component of the virion but is required to maintain the transformed cell phenotype.

Progress towards the identification and isolation of this crucial viral protein will inevitably be tantalizingly slow, but analysis of the Rous virion proteins is progressing. Rifkin and Compans (ibid., 485), for example, have now shown that the spikes, which, in the electron microscope, can be seen projecting from the surface of Rous virions, are removed by treatment with bromelain and their loss coincides with the loss of glucosamine containing proteins and of infectivity. Rifkin and Compans conclude therefore that the spikes of Rous sarcoma virions are glycoproteins necessary for infectivity and that they resemble the spikes of other enveloped viruses including flu and SV5 viruses.

\section{PROTEIN SYNTHESIS}

\section{Asserting the Fuith}

from our Molecular Biology Correspondent

From time to time a need arises within each field of research to provide the faithful with a sign. Some fifteen years since the word concerning the triplet code went forth, Gupta et al. (Biochemistry, 10, 4410; 1971) now offer more or less direct evidence that with each amino-acid incorporated into a polypeptide chain the ribosome does indeed, as everybody knew it would, slide, roll, bounce or lurch three nucleotide units along the messenger in the $5^{\prime}$ to $3^{\prime}$ direction.

Leaving out the separate processes of initiation and termination, the cycle of synthesis in bacterial systems is at present thought to go as follows: a quaternary complex is formed between a protein factor, GTP, an aminoacyltRNA and the A-binding site of the ribosome, sitting on its messenger, with the partly synthesized polypeptide chain, in the form of polypeptidyl-tRNA, at the other other (P) site. The GTP is then hydrolysed and the resulting GDP, still bound to the protein factor $\left(\mathrm{S}_{3}\right)$, as well as the orthophosphate that is formed, are liberated. The peptidyltRNA is hydrolysed, and the peptide chain makes a new peptide link with the amino-acid attached to its tRNA in the A-site. There is then a translocation step, evidently triggered by another ribosomal factor $\left(\mathrm{S}_{2}\right)$ with consumption of a further GTP, at the end of which the peptidyl-tRNA has returned to the $\mathrm{P}$ site, and the discharged tRNA has departed. Thereafter it is only necessary to regenerate a quaternary complex, a process which requires the intervention of a third factor $\left(S_{1}\right)$ to dissociate the $S_{3}-$ GDP, for the cycle to repeat. One takes it that GTP hydrolysis provides the mechanochemical urge to displace the ribosome along the messenger, but Gupta et al. point out that the implication of GTP at two points in the cycle leaves some doubt about the exact stage at which translocation occurs. They have accordingly examined the system frozen in turn at each state of the cycle.

The messenger is $\mathrm{f} 2$ phage RNA, and an initiation complex, involving ribosomes and formylmethionyl-tRNA, forms at the beginning of the coat protein cistron of the messenger. By adding or omitting the appropriate factors, $S_{1}$, $S_{2}$ and $S_{3}$, the system can be put into the pre- or post-translocation state. All of the messenger not in the ambit of the ribosome, and therefore protected by it, can then be trimmed away with nuclease. The remaining messenger can be dissociated from the ribosome, and its $3^{\prime}$ terminal sequence determined. The messenger trapped, after the digestion, in the initiation or the pre-translocation complex ends with the phenylalanine codon, UUU, corresponding to the fourth residue in the coat protein after formylmethionine. After translocation an additional triplet on the $3^{\prime}$ side is found to be protected. This is ACU,

\section{Helium in Globular Cluster Stars}

Globular clusters are of great importance because they are the oldest stellar systems in our galaxy. Thus, their ages may not only set a limit on the age of the galaxy, but may also provide an important clue to time scale over which the universe as a whole has evolved. A great deal of careful observation is necessary before such clusters can be accurately dated and determinations made of the distance to a cluster (and hence absolute luminosities), the composition of the stars in the cluster (particularly their helium and "metal" content) and the point at which the cluster members are leaving the main sequence. These data can then be related to theoretical evolutionary tracks for stars near the main sequence.

One bone of contention arising from such studies has been the exact choice of helium content necessary to fit the theoretical models to the observed properties of particular clusters. In next Monday's Nature Physical Science (January 10) John Faulkner, of the Lick Observatory, points out a convenient method for determining this from the exact shape of the evolutionary track followed by cluster members between the turn off from the main sequence and arrival at the giant branch.

Faulkner has looked at the best models now available for the cluster M 92. Between the main sequence and the giant branch, the rate of luminosity evolution of a star depends critically on its helium content, although in the main sequence and giant branch themselves composition is less important in this respect. This effect is largely independent of convection, the treatment of which is most uncertain in stellar evolution theory.

For M 92, Faulkner finds that comparison of the slope in the critical region with theoretical models of Simoda and Iben (Astrophys. J. Supp., 22, $81 ; 1970$ ) implies a helium content of $Y=0.33$. Values as low as $Y=0.2$ can be specifically excluded. This method of determining helium abundance by accurate measurement of just one portion of the evolutionary track of a cluster, rather than trying to relate all of the observations to a complete theoretical model, offers a powerful aid in the study of globular clusters. 